\title{
Efficacy of p16 and Ki-67 immunostaining in the detection of squamous intraepithelial lesions in a high-risk HPV group
}

\author{
SHARON LIM ${ }^{1}$, MI JA LEE ${ }^{1}$, INJU CHO ${ }^{1}$, RAN HONG $^{1}$ and SUNG CHUL LIM ${ }^{1,2}$ \\ ${ }^{1}$ Department of Pathology and ${ }^{2}$ Research Center for Resistant Cells, \\ College of Medicine, Chosun University, Gwangju Metropolitan City 501-759, Republic of Korea
}

Received June 19, 2014; Accepted November 10, 2015

DOI: $10.3892 / \mathrm{ol} .2015 .4071$

\begin{abstract}
Ki-67 immunohistochemistry can be used as an ancillary method for the diagnosis of squamous intraepithelial lesion (SIL) versus atrophic change and atypical squamous metaplasia. The aim of the present study was to evaluate the efficacy of these two immunohistochemical markers in the accurate interpretation of cervical biopsies and correlate this data with human papilloma virus (HPV) infection status. The study included 103 formalin-fixed cervical punch and cone biopsy samples, with corresponding HPV DNA test data. Histopathological staining with hematoxylin and eosin, and immunohistochemical staining for p16 and Ki-67 were reviewed by two pathologists. The positivity of p16 and Ki-67 increased significantly with the severity of the cervical lesion in patients with a high-risk-HPV (HR-HPV) infection status $(\mathrm{P}<0.001)$. However, there was discordance in the HPV-negative group. Furthermore, concomitant diffuse, strong and block positive staining of p16, and a high Ki-67 index were implicated in high-grade SIL in the HR-HPV group. Thus, the two markers were efficient in advancing the diagnostic accuracy of cervical biopsies in the HR-HPV group; however, application of immunohistochemical results should be carefully considered in the HPV-negative group.
\end{abstract}

\section{Introduction}

Uterine cervical cancer represents the second most common type of cancer, in terms of incidence and mortality rates (15.2 and 7.8 cases per 100,000 individuals, respectively), in women worldwide, with an increased incidence (17.8 cases per 100,000 individuals) in low resource countries (1). Almost all

Correspondence to: Professor Sung Chul Lim, Department of Pathology, College of Medicine, Chosun University, 309 Pilmun Boulevard, Dong, Gwangju Metropolitan City 501-759, Republic of Korea

E-mail: sclim@chosun.ac.kr

Key words: uterine cervix, squamous intraepithelial lesion, human papilloma virus, p16, Ki-67 cases of cervical cancer are caused by persistent infections with high-risk-human papilloma virus (HR-HPV), which may cause cervical intraepithelial neoplasia (CIN) and squamous cell carcinoma (SCC) (2).

Cervical biopsy, which is used in conjunction with Papanicolaou cytology testing, HPV DNA testing and colposcopy, has an important role in the evaluation and management of women with cervical dysplastic lesions. Thus, cervical biopsy is crucial for the prevention and early detection of cervical cancer (3). However, misinterpretation of histological changes may occur for various reasons, such as the presence of atrophy, immature metaplasia, transitional metaplasia, reparative or inflammatory atypia, inadequate sample size and tissue artifacts. These factors may cause inter-observer variation and poor intra-observer reproducibility (4-6).

A number of biomarkers have been evaluated for their potential to improve the diagnostic consistency and accuracy of cervical biopsy interpretation (4-6). Many of these studies (7-9) and other such studies $(10,11)$ endorse the use of p16 and Ki-67 immunostains, and more recently the ProExC immunostain (11-13), as useful adjunct techniques to confirm a diagnosis of high-grade squamous intraepithelial lesion (HSIL) and to distinguish it from its mimics. p16 is a cyclin-dependent kinase inhibitor and a surrogate marker for HPV E7-mediated degradation of retinoblastoma protein (pRb) $(14,15)$. There is evidence to suggest that a negative feedback mechanism controlling p16 levels in normal cells is disrupted by a reduction of $\mathrm{pRb}$ activity in proliferating squamous epithelial cells expressing HR-HPV E7 (16). With regard to p16, it has been demonstrated that almost $100 \%$ of cases of HSIL and SCC associated with HR-HPV express high levels of p16, whereas non-dysplastic cervical epithelium or low-grade SIL (LSIL) associated with low-risk (LR) or negative HPV types do not express p16 (17). Ki-67 is a nuclear protein and is detected by mindbomb E3 ubiquitin protein ligase-1 (MIB-1), a monoclonal antibody that is associated with RNA transcription and cell cycle progression (18). To improve diagnostic accuracy, other markers, including Ki-67, have been used in conjunction with p16 in the histological assessment of SIL and SCC of the uterine cervix $(5,19)$. Similar to p16, Ki-67 is overexpressed in HSIL and SCC (20).

Considering the aforementioned knowledge, the present study aimed to investigate the efficacy of p16 and Ki-67 immunohistochemical stains in the pathological assessment of 
uterine cervical biopsy samples, and identify any viral and histopathological correlations.

\section{Materials and methods}

Materials. The following terms were used to search in the pathology data program of Chosun University Hospital, (Gwangju, Republic of Korea) for the final reports of patients who had undergone HPV DNA testing of cervical biopsy specimens obtained between January 2012 and March 2013: 'Squamous metaplasia', 'LSIL' and 'HSIL'. According to the 2014 WHO classification of tumors of the female genital tract (21), a total of 103 specimens were identified with the following pathological diagnoses: Squamous metaplasia, 1 case; LSIL with HPV, 30 cases; LSIL with CIN 1, 22 cases; HSIL with CIN 2, 21 cases; and HSIL with CIN 3, 29 cases. By reviewing the medical records and pathological reports of the selected patients, details of the patients' age, underlying disease, other tumor history, cervico-vaginal cytological diagnosis and HPV infection status were identified. The present study was approved by the Institutional Review Board (IRB) of the Chosun University Hospital (Gwangju, Korea) (CHOSUN 2014-03-002). IRB approval included a waiver of informed consent.

HPV DNA testing and genotyping of HPV DNA for HR-HPV were achieved by polymerase chain reaction-based HPV genotyping assay using a HPV 9G DNA chip (Fammed Co., Ltd., Seongnam, Republic of Korea), according to the manufacturer's instructions. The DNA chip was able to detect 28 HPV genotypes, including 14 HR-HPVs (16, 18, 31, 33, 39 , 45, 51, 52, 54, 56, 58, 59, 66 and 68) and 7 LR-HPVs (6, 11, 34, 40, 42, 43 and 44). The HPV genotypes were classified as HR-HPV or LR-HPV according to the scheme proposed by Dunne et al (22).

Immunohistochemical analysis. Prior to immunohistochemical analysis, $3-\mu \mathrm{m}$ thick serial sections were prepared from representative formalin-fixed, paraffin-embedded tissue blocks and mounted on positively charged glass slides. Immunostaining was performed using a BenchMark XT autostainer (Ventana Medical Systems, Tucson, AZ, USA). The primary antibodies were anti-human p16 (mouse monoclonal antibody, clone E6H4; cat no. 725-4713; 1:100 dilution; Ventana Medical Systems) and Ki-67 (mouse monoclonal antibody, clone MM1; cat no. ACK02; 1:50 dilution; Leica Biosystems, Ltd., Newcastle, UK). Briefly, antigen retrieval was performed using peroxidase and alkaline phosphatase blocking reagent (Dako Korea LLC, Seoul, Republic of Korea) for $10 \mathrm{~min}$ at $95-99^{\circ} \mathrm{C}$ in a water bath. After inactivation of endogenous peroxidases, the slides were incubated for $30 \mathrm{~min}$ at room temperature with primary antibodies or negative control antibody. The slides were then incubated with polyclonal antimouse secondary antibody (cat. no. E0433; 1:250 dilution) for $30 \mathrm{~min}$ at room temperature. Primary antibodies and alkaline phosphatase enzyme complexes (cat. no. D0651; Dako Korea LLC) were incubated for $30 \mathrm{~min}$ at room temperature, then visualized following incubation with 3,3'-diaminobenzidine (Dako Korea LLC) for $20 \mathrm{~min}$ at room temperature. Next, the slides were counterstained with Mayer's hematoxylin (Merck Millipore, Darmstadt, Germany) for $5 \mathrm{~min}$ at room
Table I. Comparison of p16 result and final diagnosis according to HPV infection status.

\begin{tabular}{lccccc}
\hline & \multicolumn{4}{c}{ Final diagnosis, $\mathrm{n}(\%)$} & \\
\cline { 2 - 5 } HPV status & p16 & HSIL & LSIL & NILM & P-value \\
\hline HR (n=71) & + & $42(59.1)$ & $1(1.4)$ & $0(0.0)$ & $<0.001$ \\
& - & $1(1.4)$ & $26(36.6)$ & $1(1.4)$ & \\
LR (n=6) & + & $1(16.6)$ & $0(0.0)$ & $0(0.0)$ & 0.014 \\
& - & $0(0.0)$ & $5(83.3)$ & $0(0.0)$ & \\
N (n=26) & + & $5(19.2)$ & $0(0.0)$ & $0(0.0)$ & 0.001 \\
& - & $3(11.5)$ & $16(61.5)$ & $2(7.7)$ & \\
Total (n=103) & + & $48(46.6)$ & $1(0.9)$ & $0(0.0)$ & \\
& - & $4(3.9)$ & $47(45.6)$ & $3(2.9)$ & \\
\hline
\end{tabular}

HPV, human papilloma virus; HSIL, high-grade squamous intraepithelial lesion; LSIL, low-grade squamous intraepithelial lesion; NILM, negative for intraepithelial lesion or malignancy; HR, high-risk; +, positive; -, negative; LR, low-risk; N, negative.

temperature. The slides were visualized under a microscope (BX50; Olympus Corporation, Tokyo, Japan).

Immunohistochemical interpretation. p16 staining was interpreted as positive when nuclear or nuclear and cytoplasmic, strong and diffuse block staining beginning from the basal cell layer of the epithelium was observed. By contrast, non-specific focal or patch nuclear staining was considered to indicate negative p16 staining, with cytoplasmic only, wispy, blob-like, puddled, scattered, single cells and a complete lack of staining also defined as negative p16 expression (23). The expression of Ki-67 was categorized into four groups based on the distribution and proportions of cells with positive nuclear staining, as follows: Score $0,<10 \%$ of the cells, restricted to the parabasal cell layers; score 1, 10-29\% of the cells, restricted to the lower third of the epithelium; score 2, 30-69\% of the cells, reaching the upper third of the epithelium; score $3, \geq 70 \%$ epithelial cells, including full thickness expression of Ki-67 (24).

Histological evaluation. Hematoxylin and eosin staining was performed according to standard protocols (25). Two independent observers evaluated the hematoxylin and eosin (H\&E)-stained slides, and then determined initial diagnoses based on the histological features according to the 2014 WHO Classification (21). The whole slides were reviewed independently by two observers who were blinded to all clinicopathological information. These diagnoses included benign lesions, such as squamous metaplasia or LSIL, and precancerous lesions, such as HSIL. The observers used a two-tier system of terminology, according to The Lower Anogenital Squamous Terminology Standardization Project for HPV-Associated Lesions (23). Then, each observer evaluated the H\&E slides a second time, and analyzed p16 and Ki-67 staining. The final diagnosis was determined by revising all diagnoses against each other and by a final comparison of all diagnoses by a third observer. The two pathologists' diagnoses were concordant with the diagnosis of the third pathologist in all 103 cases. Finally, consensus diagnoses were reached. 
Table II. Comparison of $\mathrm{Ki}-67$ result and final diagnosis according to HPV infection status.

\begin{tabular}{|c|c|c|c|c|c|}
\hline \multirow[b]{2}{*}{ HPV status } & \multirow{2}{*}{$\begin{array}{l}\mathrm{Ki}-67 \\
\text { score }\end{array}$} & \multicolumn{3}{|c|}{ Final diagnosis, n (\%) } & \multirow[b]{2}{*}{ P-value } \\
\hline & & HSIL & LSIL & NILM & \\
\hline \multirow[t]{4}{*}{$\mathrm{HR}(\mathrm{n}=71)$} & 0 & $0(0.0)$ & $16(22.5)$ & $1(1.4)$ & \multirow[t]{4}{*}{$<0.001$} \\
\hline & 1 & $3(4.2)$ & $9(12.6)$ & $0(0.0)$ & \\
\hline & 2 & $14(19.7)$ & $2(2.8)$ & $0(0.0)$ & \\
\hline & 3 & $26(36.6)$ & $0(0.0)$ & $0(0.0)$ & \\
\hline \multirow[t]{4}{*}{$\operatorname{LR}(n=6)$} & 0 & $0(0.0)$ & $2(33.3)$ & $0(0.0)$ & \multirow[t]{4}{*}{$<0.001$} \\
\hline & 1 & $1(16.6)$ & $1(16.6)$ & $0(0.0)$ & \\
\hline & 2 & $0(0.0)$ & $2(33.3)$ & $0(0.0)$ & \\
\hline & 3 & $0(0.0)$ & $0(0.0)$ & $0(0.0)$ & \\
\hline \multirow[t]{4}{*}{$N(n=26)$} & 0 & $0(0.0)$ & $10(38.4)$ & $2(7.7)$ & \multirow[t]{8}{*}{$<0.001$} \\
\hline & 1 & $0(0.0)$ & $2(7.7)$ & $0(0.0)$ & \\
\hline & 2 & $7(26.9)$ & $1(3.8)$ & $0(0.0)$ & \\
\hline & 3 & $1(3.8)$ & $1(3.8)$ & $0(0.0)$ & \\
\hline \multirow[t]{4}{*}{ Total $(n=103)$} & 0 & $0(0.0)$ & $28(27.2)$ & $3(2.9)$ & \\
\hline & 1 & $4(3.9)$ & $14(13.6)$ & $0(0.0)$ & \\
\hline & 2 & $21(20.3)$ & $5(4.8)$ & $0(0.0)$ & \\
\hline & 3 & $27(26.2)$ & $1(0.9)$ & $0(0.0)$ & \\
\hline
\end{tabular}

HPV, human papilloma virus; HSIL, high-grade squamous intraepithelial lesion; LSIL, low-grade squamous intraepithelial lesion; NILM, negative for intraepithelial lesion or malignancy; HR, high-risk; LR, low-risk; N, negative.

Statistical analysis. SPSS software (version 22.0; SPSS, Inc., Chicago, IL, USA) was used for statistical analysis. The $\chi^{2}$ test was performed between the final diagnoses of p16 and Ki-67 status. These values were then compared with the following HPV infection statuses: HR-HPV, LR-HPV and HPV-negative. $\mathrm{P}<0.05$ was considered to indicate a statistically significant difference.

\section{Results}

\section{Expression of pl6 and $\mathrm{Ki}-67$}

HR-HPV group. The HPV genotyping assay detected HR-HPV genotypes in $68.93 \%(71 / 103)$ of cases. Of the 28 cases of benign lesion (NILM and LSIL), 27 cases were p16 negative, with only 1 case of LSIL exhibiting p16 positivity. Excluding 1 case of negative p16 expression, almost all of the 43 cases of HSIL exhibited positive expression for p16 (Table I).

There were 17 cases HR-HPV with Ki-67 negativity,including 16 cases of LSIL and 1 case of NILM. All Ki-67 staining scores of 3 in the HR-HPV group were HSIL. Twelve cases showed score of 1 in Ki-67 staining that of 3 cases of HSIL and 9 cases of LSIL. A Ki-67 score of 2 was observed in 16 cases, including 14 cases of HSIL and 2 cases of LSIL (Table II). In HR-HPV patients, p16 positivity (Table I) and Ki-67 score (Table II) were found to correlate with the severity of dysplasia (both $\mathrm{P}<0.001$ ).

LR-HPV group. The HPV genotyping assay detected 5.82\% (6/103) cases with LR-HPV genotypes. In the 5 cases of LSIL, all were p16 negative. Furthermore, there was only 1 case of HSIL with p16 positivity (Table I) and $2 / 5$ cases (33.3\%) of LSIL
Table III. Cases of discordance between HPV $\mathrm{N}$ group and result of HPV genotyping.

\begin{tabular}{lcccccc}
\hline & $\begin{array}{c}\text { Initial } \\
\text { Case }\end{array}$ & $\begin{array}{c}\text { Final } \\
\text { diagnosis }\end{array}$ & $\begin{array}{c}\text { Ki-67 } \\
\text { diagnosis }\end{array}$ & $\begin{array}{c}\text { p16 } \\
\text { score }\end{array}$ & viral Pap & Genotype \\
\hline 1 & HSIL & HSIL & - & 2 & N & N \\
2 & HSIL & HSIL & - & 2 & N & N \\
3 & HSIL & HSIL & - & 2 & N & N \\
\hline
\end{tabular}

HPV, human papilloma virus; Pap, Papanicolaou; HSIL, high-grade squamous intraepithelial lesion; -/N, negative.

exhibited a Ki-67 score of 0 . In addition, two and one cases of LSIL had Ki-67 scores of 2 and 1, respectively. There was 1 case of HSIL with a Ki-67 score of 1 (Table II). Furthermore, in the LR-HPV group, p16 positivity (Table I) and Ki-67 score (Table II) were significantly associated with malignant potential $(\mathrm{P}=0.014$ and $\mathrm{P}<0.001$, respectively).

HPV-negative group. The HPV genotyping assay detected $25.24 \%(26 / 103)$ cases with negative HPV genotypes. All 18 cases of benign lesion (NILM and LSIL) were p16 negative. Excluding 3 cases, 5/8 cases (19.2\%) of HSIL showed positive expression for p16 (Table I). A Ki-67 score of 0 was exhibited by $12 / 18$ cases of benign lesion, including of 2 cases of NILM and 10 cases of LSIL. Furthermore, 4/16 cases of LSIL showed a Ki-67 expression score of 1 , while only 1/16 cases of LSIL exhibited a Ki-67 expression score of 2 and 3. There were 8 cases of HSIL, with a score of 3 in 1 case and a score of 2 in 7 cases (Table II). In the HPV-negative group, p16 positivity (Table I) and Ki-67 score (Table II) were significantly associated with the degree of dysplasia $(\mathrm{P}=0.001$ and $\mathrm{P}<0.001$, respectively).

Discordance between p16 and Ki-67 expression, and the HPV genotype. Analysis of the H\&E slides only revealed 2 cases of immature squamous metaplasia with an initial diagnosis of LSIL $(n=1)$ and HSIL $(n=1)$. These cases exhibited immunohistochemical negativity for p16 and a low Ki-67 index (Fig. 1). However, discordance was identified in the HPV-negative group (Table I). In the HPV-negative group, 3/26 cases exhibited completely negative expression for p16; thus, the discordance rate was $11.54 \%$. However, these cases were consistent with a pathological diagnosis of HSIL, according to the H\&E slides and the high Ki-67 index (score, 2) (Fig. 2A-C). Therefore, these cases were finally diagnosed based on histological findings and the Ki-67 result (Table III). In the LR-HPV and HR-HPV groups, expression of p16 and Ki-67 exhibited a strong correlation with malignant potential of the lesion (Fig. 2D-F). Only one case of HSIL diagnosis was negative in p16 result and one case of LSIL was positive for p16 positive in the total HR-HPV group $(n=71)$, thus, the discordant rate was $2.82 \%$ (Table I).

\section{Discussion}

Diagnostic interpretation of dysplasia in the uterine cervix typically includes analysis of hypercellularity, significant atypia, mitotic figures and disorientation from the parabasal to upper layers (21). However, unusual histological features, including 

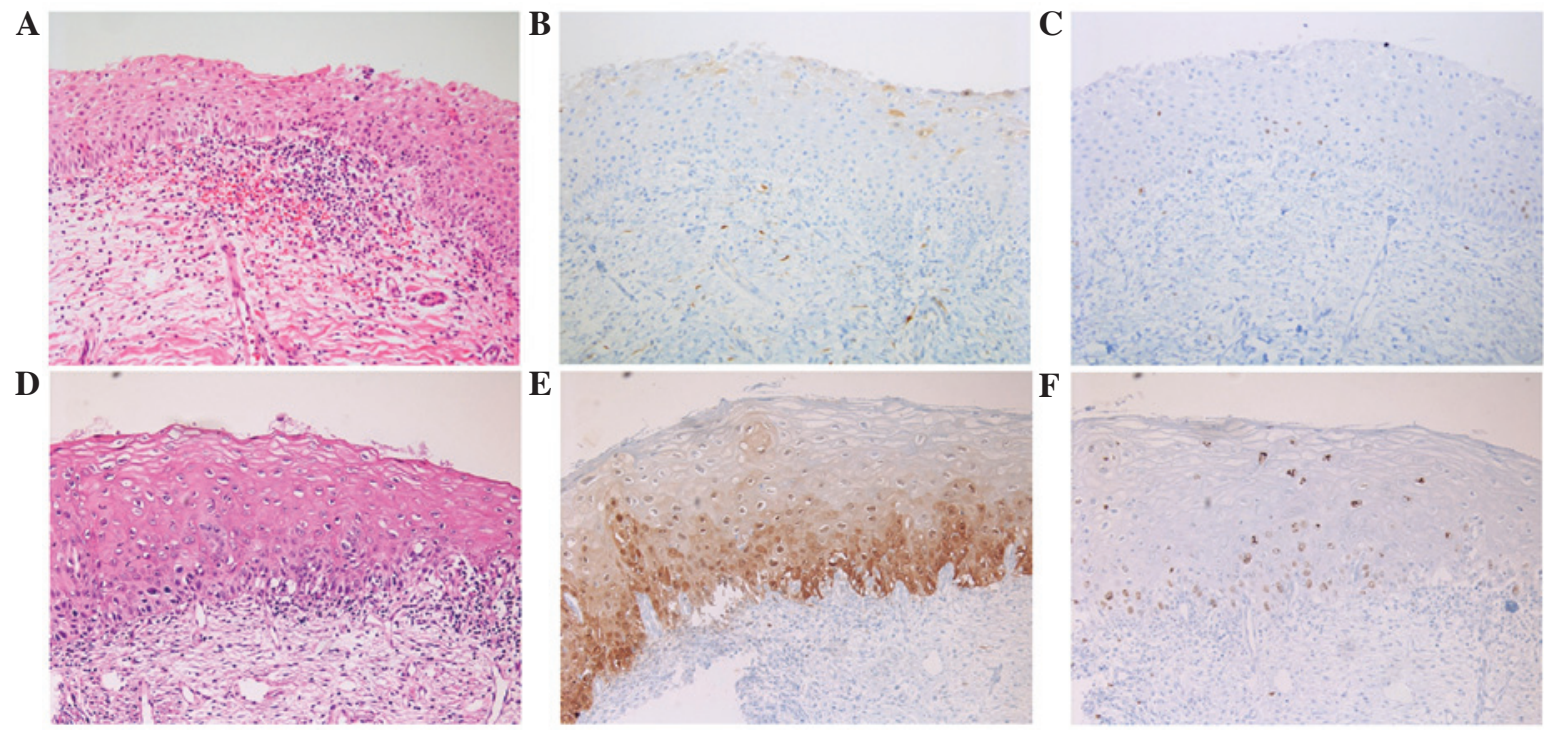

Figure 1. Upper row shows immature squamous metaplasia. (A) Hematoxylin and eosin staining. Immunohistochemical staining, revealing (B) negative p16 expression and (C) a low Ki-67 (score 0). Lower row shows low-grade squamous intraepithelial lesion. (D) Hematoxylin and eosin staining. Immunohistochemical staining, revealing (E) a patch stain for p16 in the parabasal layer that was interpretated as negative and (F) increased expression of Ki-67 (score 1) in the lower third area. Magnification, x200.
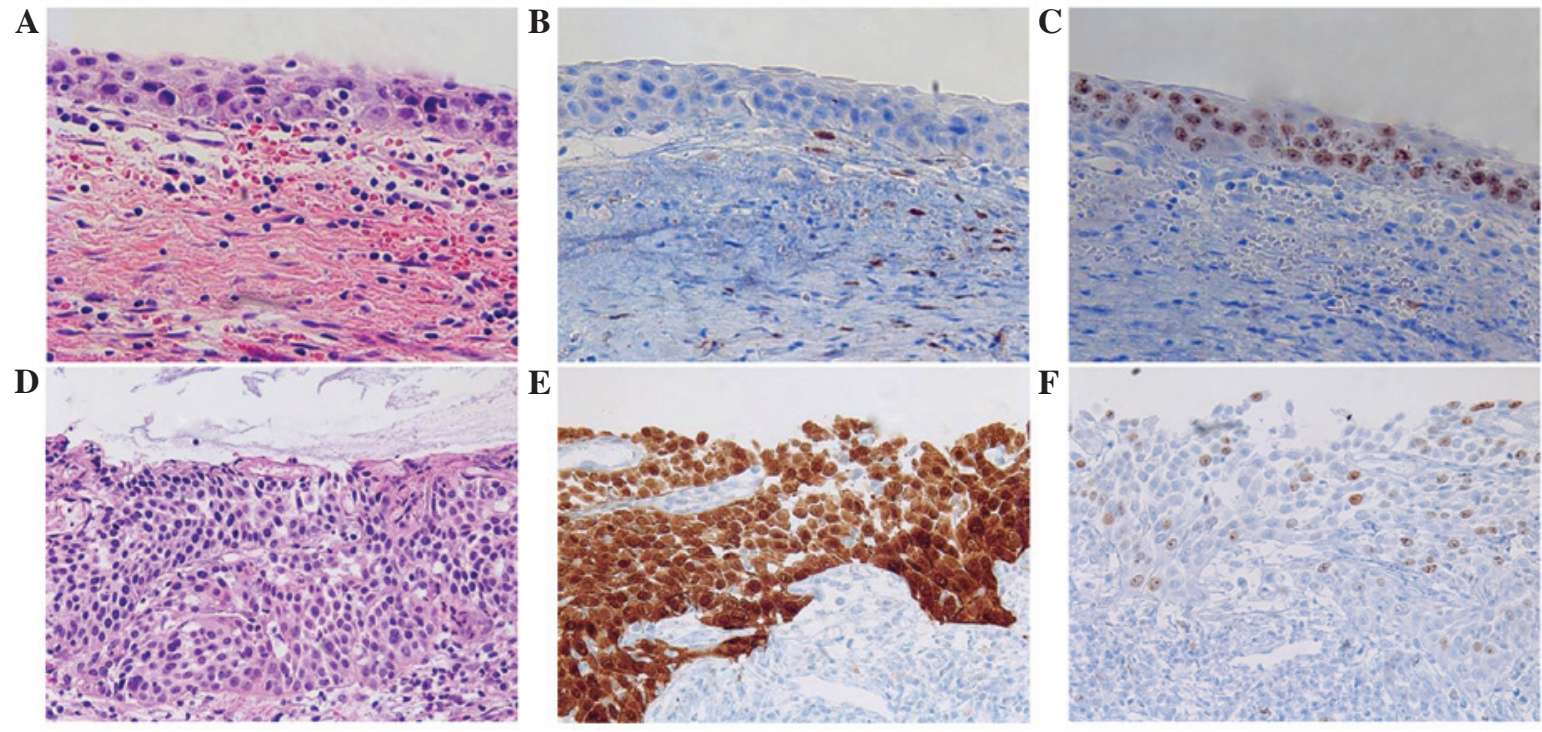

Figure 2. Upper row shows a high-grade squamous intraepithelial lesion (HSIL) in the human papilloma virus (HPV)-negative group. (A) Hematoxylin and eosin staining. Immunohistochemical staining, revealing (B) negative staining for p16 and (C) increased expression of Ki-67 (score 3) in the full thickness of the upper third area. Lower row shows HSIL in the high-risk-HPV group. (D) Hematoxylin and eosin staining. Immunohistochemical staining, revealing (E) strong block positivity of p16 and (F) an increase in Ki-67 expression in the full thickness of the sample. Magnification, x200.

mildly increased cellularity, the absence of mitotic figures and questionable atypia, may be observed in the lesion (9). In daily practice, the accurate diagnosis of cervical dysplastic lesions is subject to inter-observer and intra-observer variability by these factors (6).

Immunostaining may improve the diagnostic reproducibility and accuracy of the CIN lesion. Previous studies have demonstrated that p16 and Ki-67 are co-expressed in almost $100 \%$ of cases of high-grade squamous and glandular lesions, and these markers are rarely co-expressed in normal or benign lesions of cervical epithelial lesion (14,26-29). Immunohistochemical staining for p16 has been investigated in cervical pathology as a marker for HPV-transformed lesions, and various studies have demonstrated diffuse and continuous staining of p16, beginning in the basal and parabasal cell layers, which is defined as block positive staining $(7,23,30)$. Based on the concept that HPV-mediated transformation is triggered by dysregulated expression of the viral oncogenes E6 and E7 in basal and parabasal cells, p16 immunohistochemistry was hypothesized to distinguish between transforming and nontransforming HPV infections, and only the block positive p16 expression pattern was defined as a hallmark of HPV-dependent transformation and thus considered as p16 positive $(27,31,32)$. 
Ki-67 is detected by the MIB-1 monoclonal antibody and is a nuclear protein that is associated with RNA transcription and cell cycle progression (18). Similar to p16, Ki-67 is overexpressed in CIN 2/3, SCC, adenocarcinoma in situ and adenocarcinoma (20). However, in contrast to p16, Ki-67 is also overexpressed in the basal cells of normal squamous mucosa and in benign proliferative lesions, including basal cell hyperplasia of the squamous mucosa (26). Therefore, a combination of p16 and Ki-67 immunostaining is recommended for specificity in distinguishing LSIL versus HSIL from its mimickers, as opposed to using each immunostaining marker alone (11).

By conducting a review of previous studies, it was found that these immunostaining markers could be applied in selective HR-HPV groups and high-grade dysplastic lesions (29,30). Thus, the present study investigated the efficacy of p16 and Ki-67 immunostaining markers in the accurate interpretation of cervical biopsies, and comparing the results of HR-HPV, LR-HPV and HPV-negative groups. Diffuse and strong staining for p16 and Ki-67 revealed HSIL in the HR-HPV and LR-HPV groups. Almost all HSIL cases in the HR-HPV group were p16 positive. Obvious expression of p16 has been associated HR-HPV infection (5); this was also found in the present study. In addition, 2 cases of immature squamous metaplasia with previous LSIL $(n=1) / \operatorname{HSIL}(n=1)$ were identified by $\mathrm{H} \& \mathrm{E}$ staining alone; furthermore, these cases demonstrated negativity for p16 and a low Ki-67 index in the HR-HPV and HPV-negative viral test groups. However, numerous cases in the HPV-negative group exhibited discordancy between the H\&E and immunohistochemical findings. Three unusual cases that showed an unexpected p16 immunostaining result were identified out of the total 26 HPV-negative patients (Table III). Representatively, 2 cases were implicated pathological significance. Case 3 (Table III) demonstrated p16 negativity and a Ki-67 score of 3, and exhibited histopathological features consistent with HSIL. Thus, a final diagnosis of HSIL was determined. However, the patient's clinical stage was advanced; therefore, the therapeutic options were limited. The patient underwent radiation therapy and chemotherapeutic regimen two times but succumbed due to pancytopenia and multiple lymph node metastasis 9 months later. Case 2 (Table III) was initially diagnosed with HSIL, although the patient's immunohistochemical profile of p16 was not compatible with HSIL. However, H\&E staining and $\mathrm{Ki}-67$ index results indicated HSIL and thus, the final diagnosis was confirmed by conization specimens resected with a clear resection margin 1 month later. Case 1 (Table III) had an initial diagnosis of HSIL with negative p16 expression and a Ki-67 score of 2; in this case the first diagnosis was not revised. The diagnosis was finally confirmed by analysis of a hysterectomy specimen 3 weeks later. Three months after hysterectomy, case 1 exhibited no evidence of local recurrence or metastasis. The aforementioned 3 cases of the negative-HPV group were diagnosed via different diagnostic methods, due to the unusual p16 pathology results. Therefore further treatments, including conization and hysterectomy were performed to allow for additional pathological examinations, that did not rely on the atypical p16 scores. In HPV negative patients, we hypothesize that HSIL with a negative p16 score must be considered when interpreting cervical biopsies to prevent underdiagnosis to LSIL or NILM. For case 1 , the negative p16 result did not correspond with the clinicopathological setting, and thus H\&E staining and $\mathrm{Ki}-67$ index were applicable. It is currently unknown what causes true-negative p16 immunostaining in HSIL or SCC in the HPV-negative group. The positivity of p16 expression and the $\mathrm{Ki}-67$ score increased with the severity of the cervical lesion in the HR-HPV and LR-HPV groups. However, correlation between p16 score and final diagnosis was stronger in the HR-HPV group compared with the HPV-negative group. Thus, Ki-67 score is a more useful marker than p16 score in HPV-negative cases.

In conclusion, simultaneously positive p16 expression and high $\mathrm{Ki}-67$ index are implicated in diagnosis of HSIL for HR-HPV and LR-HPV precancerous lesions. The two markers are efficient in advancing the diagnostic accuracy of cervical biopsies in cases of HR-HPV and LR-HPV, however, application in discrete daily sign-out processing of immunohistochemical findings should be considered in the HPV-negative group. This is a pilot study with a small number of cases, however, pathologists should be aware that unusual immunostaining results in HPV-negative patients, such as negative p16 staining in HSIL, may imply factors other than HR-HPV and LR-HPV infection. In the future, novel immunostaining markers or other methods that may be applicable for HPV-negative patients should be assessed for the reproducible diagnosis of cervical disease.

\section{Acknowledgements}

The current study was supported by a National Research Foundation of Korea Grant funded by the Ministry of Education, Science and Technology (MEST) through the Research Center for Resistant Cells (grant no. R13-2003-009).

\section{References}

1. Ferlay J, Shin HR, Bray F, Forman D, Mathers C and Parkin DM: Estimates of worldwide burden of cancer in 2008: GLOBOCAN 2008. Int J Cancer 127: 2893-2917, 2010.

2. Schiffman M, Castle PE, Jeronimo J, Rodriguez AC and Wacholder S: Human papillomavirus and cervical cancer. Lancet 370: 890-907, 2007.

3. Wright TC Jr, Massad LS, Dunton CJ, Spitzer M, Wilkinson EJ and Solomon D; 2006 American Society for Colposcopy and Cervical Pathology-sponsored Consensus Conference: 2006 consensus guidelines for the management of women with cervical intraepithelial neoplasia or adenocarcinoma in situ. J Low Genit Tract Dis 11: 223-239, 2007.

4. Wright TC Jr, Cox JT, Massad LS, Twiggs LB and Wilkinson EJ; ASCCP-Sponsored Consensus Conference: 2001 Consensus guidelines for the management of women with cervical cytological abnormalities. JAMA 287: 2120-2129, 2002.

5. Kalof AN and Cooper K: p16INK4a immunoexpression: surrogate marker of high-risk HPV and high-grade cervical intraepithelial neoplasia. Adv Anat Pathol 13: 190-194, 2006.

6. Klaes R, Benner A, Friedrich T, Ridder R, Herrington S, Jenkins D, et al: p16INK4a immunohistochemistry improves interobserver agreement in the diagnosis of cervical intraepithelial neoplasia. Am J Surg Pathol 26: 1389-1399, 2002.

7. Horn LC, Reichert A, Oster A, Arndal SF, Trunk MJ, Ridder R, et al: Immunostaining for p16INK4a used as a conjunctive tool improves interobserver agreement of the histologic diagnosis of cervical intraepithelial neoplasia. Am J Surg Pathol 32: 502-512, 2008.

8. Ma L, Fisk JM, Zhang RR, Ulukus EC, Crum CP and Zheng W: Eosinophilic dysplasia of the cervix: a newly recognized variant of cervical squamous intraepithelial neoplasia. Am J Surg Pathol 28: 1474-1484, 2004 
9. Aoyama C, Liu P, Ostrzega $\mathrm{N}$ and Holschneider $\mathrm{CH}$ : Histologic and immunohistochemical characteristic of neoplastic and nonneoplastic subgroups of atypical squamous lesions of the uterine cervix. Am J Clin Pathol 123: 699-706, 2005.

10. Benevolo M, Mottolese M, Marandino F, Vocaturo G, Sindico $\mathrm{R}$, Piperno $\mathrm{G}$, et al: Immunohistochemical expression of p16INK4A is predictive of HR-HPV infection in cervical low-grade lesions. Mod Pathol 19: 384-391, 2006.

11. Pinto AP, Schlecht NF, Woo TY, Crum CP and Cibas ES: Biomarker (ProExC, p16INK4A, and MiB-1) distinction of high-grade squamous intraepithelial lesion from its mimics. Mod Pathol 21: 1067-1074, 2008

12. Sanati S, Huettner P and Ylagan LR: Role of ProExC: a novel immunoperoxidase marker in the evaluation of dysplastic squamous and glandular lesions in cervical specimens. Int J Gynecol Pathol 29: 79-87, 2010.

13. Walts AE and Bose S: p16, Ki-67, and BD ProExC immunostaining: a practical approach for diagnosis of cervical intraepithelial neoplasia. Hum Pathol 40: 957-964, 2009.

14. Cameron RI, Maxwell P, Jenkins D and McCluggage WG: Immunohistochemical staining with MIB1, bcl2 and p16 assists in the distinction of cervical glandular intraepithelial neoplasia from tubo-endometrial metaplasia, endometriosis and microglandular hyperplasia. Histopathology 41: 313-321, 2002.

15. Doorbar J, Quint W, Banks L, Bravo IG, Stoler M, Broker TR, et al: The biology and life-cycle of human papillomaviruses. Vaccine 30 (Suppl 5): F55-F70, 2012.

16. Khleif SN, DeGregori J, Yee CL, Otterson GA, Kaye FJ, Nevins JR and Howley PM: Inhibition of cyclin D-CDK4/CDK6 activity is associated with an E2F-mediated induction of cyclin kinase inhibitor activity. Proc Natl Acad Sci USA 93: 4350-4354, 1996.

17. Guo M, Baruch AC, Silva EG, Jan YJ, Lin E, Sneige N and Deavers MT: Efficacy of p16 and ProExC immunostaining in the detection of high-grade cervical intraepithelial neoplasia and cervical carcinoma. Am J Clin Pathol 135: 212-220, 2011.

18. Hitchcock CL: Ki-67 staining as a means to simplify analysis of tumor cell proliferation. Am J Clin Pathol 96: 444-446, 1991.

19. McCluggage WG: Immunohistochemistry as a diagnostic aid in cervical pathology. Pathology 39: 97-111, 2007.

20. Cina SJ, Richardson MS, Austin RM and Kurman RJ: Immunohistochemical staining for Ki-67 antigen, carcinoembryonic antigen, and p53 in the differential diagnosis of glandular lesions of the cervix. Mod Pathol 10: 176-180, 1997.

21. Reich O, Regauer S, Marth C, Schmidt D, Horn LC, Dannecker C, Menton M and Beckmann MW: Precancerous lesions of the cervix vulva and vagina according to the 2014 WHO classification of tumors of the female genital tract. Geburtshilfe Frauenheilkd 75 1018-1020, 2015.
22. Dunne EF, Unger ER, Sternberg M, McQuillan G, Swan DC, Patel SS and Markowitz LE: Prevalence of HPV infection among females in the United States. JAMA 297: 813-819, 2007.

23. Darragh TM, Colgan TJ, Cox JT, Heller DS, Henry MR, Luff RD, et al: The Lower Anogenital Squamous Terminology Standardization Project for HPV-Associated Lesions: Background and consensus recommendations from the College of American Pathologists and the American Society for Colposcopy and Cervical Pathology. J Low Genit Tract Dis 16: 205-242, 2012.

24. Reuschenbach M, Seiz M, von Knebel Doeberitz C, Vinokurova S, Duwe A, Ridder R, et al: Evaluation of cervical cone biopsies for coexpression of p16INK4a and Ki-67 in epithelial cells. Int J Cancer 130: 388-394, 2012.

25. Kiernan JA (ed): Histological and Histochemical Methods: Theory and Practice. Vol 12. 3rd edition. Butterworth-Heinemann, Oxford, UK, 1999.

26. Agoff SN, Lin P, Morihara J, Mao C, Kiviat NB and Koutsky LA: p16INK4a expression correlates with degree of cervical neoplasia: a comparison with Ki-67 expression and detection of high-risk HPV types. Mod Pathol 16: 665-673, 2003.

27. Liang J, Mittal KR, Wei JJ, Yee H, Chiriboga L and Shukla P: Utility of p16INK4a, CEA, Ki67, P53 and ER/PR in the differential diagnosis of benign, premalignant, and malignant glandular lesions of the uterine cervix and their relationship with Silverberg scoring system for endocervical glandular lesions. Int J Gynecol Pathol 26: 71-75, 2007.

28. Pavlakis K, Messini I, Athanassiadou S, Kyrodimou E, Pandazopoulou A, Vrekoussis T and Stathopoulos EN: Endocervical glandular lesions: a diagnostic approach combining a semiquantitative scoring method to the expression of CEA, MIB-1 and p16. Gynecol Oncol 103: 971-976, 2006.

29. Qiao X, Bhuiya TA and Spitzer M: Differentiating high-grade cervical intraepithelial lesion from atrophy in postmenopausal women using Ki-67, cyclin E, and p16 immunohistochemical analysis. Low Genit Tract Dis 9: 100-107, 2005.

30. Bergeron C, Ordi J, Schmidt D, Trunk MJ, Keller T and Ridder R; European CINtec Histology Study Group: Conjunctive p16INK4a testing significantly increases accuracy in diagnosing high-grade cervical intraepithelial neoplasia. Am J Clin Pathol 133: 395-406, 2010.

31. Doeberitz Mv and Vinokurova S: Host factors in HPV-related carcinogenesis: cellular mechanisms controlling HPV infections. Arch Med Res 40: 435-442, 2009

32. von Knebel Doeberitz M: New markers for cervical dysplasia to visualise the genomic created by aberrant oncogenic papillomavirus infections. Eur J Cancer 38: 2229-2242, 2002. 